tive, Catherine Lien Jensen and Lars Swanstrøm, both of the Norwegian Registration Authority, noted that "proactive information sharing between regulators could be fairly uncomplicated in theory, but it seems that differences in systems and culture make the process more complicated and difficult than many may have expected."

Moreover, efforts to promote more regulatory cooperation have been hampered by the refusal of 8 member states - Bulgaria, the Czech Republic, Greece, Latvia, Lithuania, Luxembourg, Romania and Slovakia - to participate.

A study by the World Health Organization, Assuring the Quality of Healthcare in the European Union: A Case for Action, suggests that 6 of the 8 have dodged participation because they "do not have any legislation on quality of care, or national policies on quality." As well, some nonparticipants may be leery of involvement in what is seen as an European Union organ or administrative body.

The Czech Republic and Lithuania have only recently implemented national legislation to control health care quality. The WHO study concludes that, in Lithuania, "the majority of measures envisaged have not been implemented due to lack of funds."

Others argue that many of the problems are associated with the current system for transferring certification between countries.

At present, doctors within the European Union are required to obtain a Certificate of Current Professional Status, which addresses both current status and past training. Complaints arise, the WHO stated, "because the criteria for recognition relate almost exclusively to the length of study, with no consideration of the content."

Despite such concerns, many believe increased mobility helps elevate all systems in the final analysis. As Maissoneuve says, all the movement means "greater competition, which is not absolutely negative, in that it pushes health care systems themselves to adapt and compete." - Ira Allen, Paris, France

DOI:10.1503/cmaj.081817

\title{
Colour-coded wristbands confusing
}

$\mathrm{T}$ hink of them as a form of hospital shorthand. Commonly used in American hospitals to instantly communicate conditions of patient care, colour-coded hospital wristbands were becoming the norm to signify allergies or do-not-resuscitate orders.

The problem was, in some states, as many as 6 or 7 colours were being used to indicate the same condition, so health care professionals working in multiple settings needed institutional colour charts just to do their jobs.

In a bid to constrain the proliferation American Hospital Association has stepped into the colour fray to simplify and streamline the range of wristband options and thus reduce the likelihood of caregiver error.

The Association has asked all hospitals to pare their list of wristbands to a bare minimum of 3: red for allergy, yellow for fall risk and purple for do not resuscitate.

Standardization of wristband colours has become essential, says American Hospital Association Vice-President Media Alicia Mitchell. "State hospital associations had already taken the lead in trying to standardize these colours. ... It only made sense for us to step in and put together these voluntary guidelines."

The situation was rapidly spiralling out of control, particularly when comand eliminate medical mix-ups, the

bined with fashion trends such as "livestrong" bracelets (yellow silicone wristbands popularized by former Tour de France cycling champion Lance Armstrong to promote cancer awareness), Mitchell adds. It can lead doctors and nurses to make incorrect conclusions about patient needs or preferences and only confuse the situation further.

Some 20 states have already indicated they will adopt the 3-colour guidelines, although many also expanded their colour palate to cover other data, such as white for patient identification or green for patients who are receiving blood products.

Canadian officials say there's no need to move with similar national standards because wristbands are rarely used in Canada as indicators of specific care needs.

"Some facilities may use a red or redstriped wristband to indicate allergies," says Teresa Neuman, communications specialist for the Canadian Healthcare Association. "Other facilities, however, do not [use wristbands] in consideration of patient privacy and confidentiality."

Relevant patient information, such as fall risk or do-not-resuscitate preferences, Neuman adds, is more typically indicated on patient charts in Canada. - Lesley Ananny, Ottawa, Ont.

\section{DOI:10.1503/cmaj.081818}

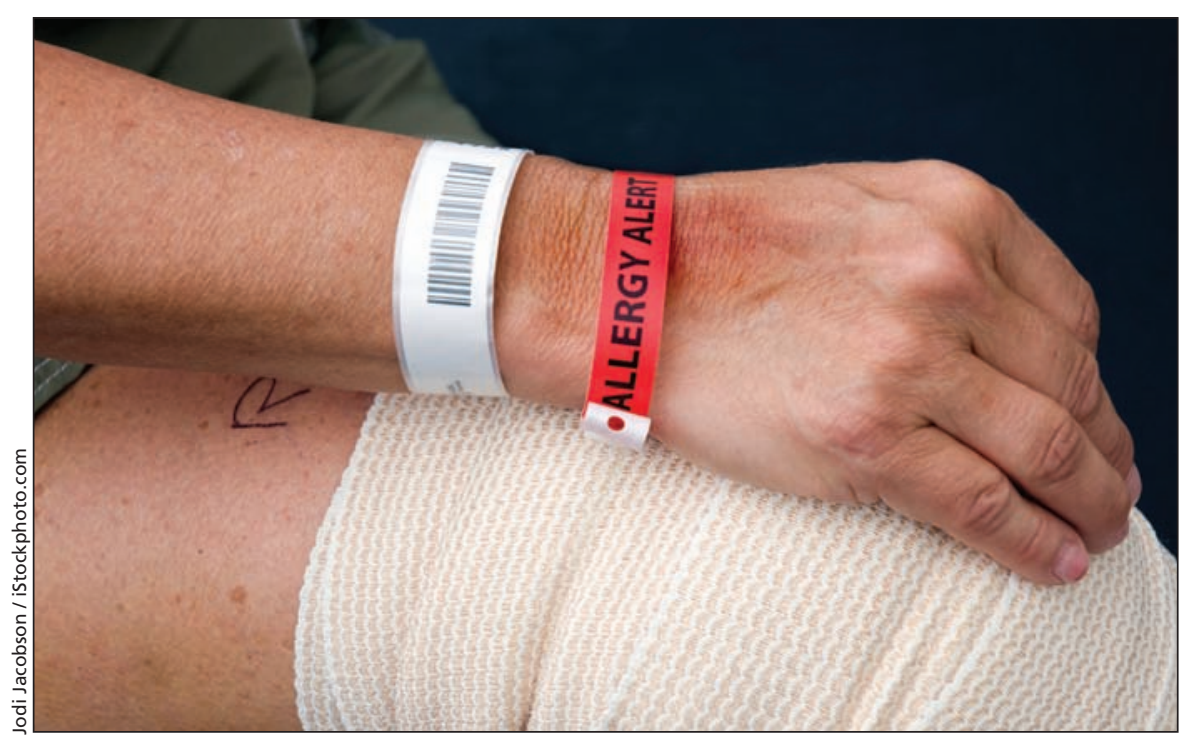

The American Hospital Association is seeking standardization of colour-coded wristbands. 